\title{
A Theory of Visual Information Acquisition and Visual Memory With Special Application to Intensity-Duration Trade-Offs
}

\author{
Geoffrey R. Loftus and Eric Ruthruff
}

\begin{abstract}
We describe a theory of memory for visual material in which the visual system acts as a linear filter operating on a stimulus to produce a function, $a(t)$, relating some sensory response to $t$ (the time since stimulus onset). Stimulus information is acquired at a rate proportional to the product of the magnitude by which $a(t)$ exceeds some threshold, and the amount of as-yet-unacquired information. Recall performance is assumed to equal the proportion of acquired information. The theory accounts for data from 2 digit-recall experiments in which stimulus temporal waveform was manipulated. We comment on the theory's account of the relation between 2 perceptual events: the phenomenological experience of the stimulus, and the memory representation that accrues from stimulus presentation. We assert that these 2 events, although influenced by different variables, can be viewed as resulting from 2 characteristics of the same sensory-response function.
\end{abstract}

In this article, we discuss the perception of and memory for complex visual stimuli. By complex we mean stimuli (such as alphanumeric characters, words, or naturalistic scenes) that must be pattern-recognized, interpreted, and processed by the cognitive system rather than simple, to-bedetected light patches or sine-wave gratings. Our major goal is to incorporate a model that has been successfully used to account for a variety of low-level visual phenomena (e.g., time-intensity trade-offs and flicker perception) into a broader theory designed to account for higher level cognitive tasks such as recall and picture recognition.

Our theory development is motivated in part by the substantial body of research implying intimate perceptual connections between stimulus duration on the one hand and stimulus intensity on the other. Before describing the theory we provide a brief sketch of this research and describe an experiment designed to relate it to memory for visual material.

\section{Bloch's Law: Strong Intensity-Duration Trade-Offs With Simple Visual Stimuli}

The most fundamental duration-intensity relation is captured in Bloch's Law, which asserts that for stimuli shorter

Geoffrey R. Loftus, Department of Psychology, University of Washington; Eric Ruthruff, Department of Psychology, University of California, San Diego.

This research was supported by National Institute of Mental Health Grant MH41637 to Geoffrey R. Loftus. We thank John Hogben, Dave Irwin, and an anonymous reviewer for very helpful comments on a draft of the article. Although they should not be blamed for any logical or factual lapses, George Sperling, Brian Wandell, and Beau Watson provided very useful advice and comments on the research in general.

Correspondence concerning this article should be addressed to Geoffrey R. Loftus, Department of Psychology, University of Washington, Seattle, Washington 98195 . Electronic mail may be sent to gloftus@u.washington.edu. than some critical duration (around $100 \mathrm{~ms}$ ) there is an almost perfect trade-off between intensity and duration with respect to threshold detection performance. Essentially, detection performance in the critical range depends only on the integral of intensity, irrespective of how this intensity has been distributed over time. Bloch's Law has been confirmed many times, but almost always within the context of simple stimuli and simple tasks. ${ }^{1}$

\section{Weaker Intensity-Duration Trade-Offs With Complex Visual Stimuli}

Does Bloch's Law apply within the context of more complex stimuli and memory tasks rather than threshold detection tasks? An affirmative answer would set the stage for extending models originally designed to account for lowlevel perceptual tasks in such a way as to account for higher level cognitive tasks as well.

The answer to this question is thus far unclear. Memory for complex visual stimuli has been found to improve with greater stimulus duration (e.g., Loftus \& Kallman, 1979; Potter \& Levy, 1969; Shaffer \& Shiffrin, 1972) and also with greater stimulus intensity (e.g., Loftus, 1985; Loftus, Kaufman, Nishimoto, \& Ruthruff, 1992). However, the only exact measurement of the duration-intensity relation using complex stimuli in a memory task was reported by Turvey (1973), who found a data pattern conforming to Bloch's Law with respect to memory for digit trigrams in a backward masking paradigm. Provocative though it was, this finding was ancillary to Turvey's major goals (which principally involved an empirical and theoretical investigation of masking); furthermore, Turvey's duration and intensity ranges were limited.

\footnotetext{
'Instances of these confirmations are Kahneman (1968), Kahneman and Norman (1964), Kaswan and Young (1963), Raab and Fehrer (1962), and Zacks (1970). Reviews are provided by Watson (1986) and Wasserman and Kong (1979, plus associated commentaries).
} 
Loftus (1985; Loftus, Kaufman, et al., 1992; see also Sperling, 1986) tested subjects in both short-term and long-term memory tasks and found evidence for a weak form of intensity-duration trade-off with complex pictures. Specifically, lowering intensity required that duration be increased by some factor $k$ (referred to as the "slowdown factor") to achieve some criterion memory performance. Within certain boundary conditions, the slowdown factor was independent of the particular criterion performance level that was chosen; that is, the relation between intensity, $\phi$, and duration, $d$, could be described by

$$
p\left(d, \phi_{1}\right)=p\left(k d, \phi_{2}\right)
$$

where $p(\mathrm{x}, \mathrm{y})$ is performance for a stimulus of duration $\mathrm{x}$ and intensity $\mathrm{y}, \phi_{1}>\phi_{2}$, and $k>1.0$. This finding is consistent with the proposition that decreasing intensity simply slows down perceptual processing by the slowdown factor, $k$, without otherwise affecting the system.

The multiplicative relation between intensity and duration implied by Equation 1 is necessary, but not sufficient, to infer a simple Bloch's Law relation in a relatively complex memory task. To show that Bloch's Law held would require determining that in Equation 1, $\phi_{1}=k \phi_{2}$. However, no experiment to date provides the data needed to draw that conclusion. In some relevant experiments, the stimuli were complex, naturalistic color photographs. Here, each stimulus included a wide range of colors and intensities, and stimulus intensity could not be precisely measured; thus, only qualitative conclusions were possible, namely, the less intense the stimuli, the greater the slowdown factor (Loftus, 1985; Loftus, Kaufman, et al., 1992). In other experiments the complete intensity/slowdown function could not be characterized either because the range of stimulus durations and the range of stimulus intensities were too small, or because the use of a backward mask obscured conclusions made purely on the basis of duration and intensity (e.g., Turvey, 1973; see Eriksen, 1980, for a discussion of the problems associated with basing conclusions on data from experiments in which

Table 1

Summary of Background Luminances, Foreground (Digit) Luminances, Contrasts, and Intensities for Each of the Four Observers

\begin{tabular}{|c|c|c|c|c|}
\hline & \multicolumn{4}{|c|}{ Intensity level } \\
\hline & 1 & 2 & 3 & 4 \\
\hline \multicolumn{5}{|c|}{ Observers C.A. and K.G. } \\
\hline Background & 23.961 & 25.200 & 27.531 & 31.689 \\
\hline Digits & 22.218 & 22.680 & 22.890 & 24.423 \\
\hline Contrast & 0.038 & 0.053 & 0.092 & 0.129 \\
\hline Intensity & 0.904 & 1.326 & 2.534 & 4.103 \\
\hline \multicolumn{5}{|c|}{ Observers G.L. and E.R. } \\
\hline Background & 25.200 & 27.531 & 31.689 & 37.926 \\
\hline Digits & 22.680 & 22.890 & 24.423 & 24.927 \\
\hline Contrast & 0.053 & 0.092 & 0.129 & 0.207 \\
\hline Intensity & 1.326 & 2.534 & 4.103 & 7.844 \\
\hline
\end{tabular}

Note. Luminances and intensities are in candelas per square meter. Intensity levels for observers C.A. and K.G. ranged from 0.90 to $4.10 \mathrm{~cd} / \mathrm{m}^{2}$; for observers G.L. and E.R., they ranged from 1.33 to $7.84 \mathrm{~cd} / \mathrm{m}^{2}$ a mask is used). We carried out Experiment 1 to fill this empirical void.

\section{Experiment 1: Measurement of Duration-Intensity Trade-Offs in a Digit-Recall Paradigm}

Experiment 1 was designed to allow precise measurement of the function relating the slowdown factor to stimulus in tensity in a memory task. We used simple black-on-white digit arrays as stimuli, along with a relatively wide range of both duration and intensity. ${ }^{2}$ Both duration and intensity were precisely controlled.

\section{Method}

The basic procedure consisted of a series of trials. On each trial, a four-digit string was presented for some exposure duration on the order of 5-150 ms. The observer's task was to immediately report as many of the digits as possible in their correct positions, guessing if necessary.

Observers. Four observers participated in the experiment: ourselves (G.L. and E.R), an undergraduate student (K.G.), and a graduate student (C.A.). All observers were familiar with the purposes of the experiment. All observers were highly practiced, having participated in a minimum of 3,000 practice trials prior to beginning the experiment.

Stimuli and apparatus. Stimuli were prepared as $35-\mathrm{mm}$ slides. A stimulus consisted of a 4 (columns) $\times 3$ (rows) array of black digits on a white background. Eighty such stimuli were prepared and used repeatedly. The $4 \times 3 \times 80=960$ digits composing all stimuli were selected randomly and without replacement from the set of 10 digits. Each digit subtended a visual angle of $0.56^{\circ}$ vertically and $0.28^{\circ}$ horizontally. Digits were separated by $0.37^{\circ}$ vertically and $0.74^{\circ}$ horizontally. On a given experimental trial, one 4-digit row of one stimulus was the to-be-reported target. Target row was blocked over trials; accordingly, an observer always knew which row was the target.

Intensity control was accomplished by attenuating stimulus luminance using a Wrattan neutral-density filter. Each observer viewed stimuli at four different intensity levels. For two observers (G.L. and E.R.) intensity ranged from 1.33 to $7.84 \mathrm{~cd} / \mathrm{m}^{2}$. The other two observers (C.A. and K.G.) were substantially better at the task; to avoid ceiling performance, their intensity levels were lower, ranging from 0.90 to $4.10 \mathrm{~cd} / \mathrm{m}^{2}$. A summary of luminances, contrasts, and intensities for the four observers is provided in Table 1.

All stimuli were displayed using Kodak projectors equipped with Gerbrands tachistoscopic shutters. A random-access projector was used to display the stimuli, while standard carousel projectors were used to present a constant, uniform adapting field and a fixation point that initiated each trial. Responses were made on a numeric

\footnotetext{
${ }^{2}$ There are numerous ways of defining intensity. We defined "contrast" as the difference between background and foreground luminance divided by the sum of background and foreground luminance. Intensity was then defined to be the product of contrast and background luminance. This definition was appropriate to the nature of our display apparatus, which, unfortunately, did not allow contrast variation without concommitant variation in background intensity. Other definitions of intensity (or simply using contrast rather than intensity) did not materially change our results or our conclusions.
} 
keypad. All display equipment was enclosed in a soundproof box. All display and response collection was under the control of an AT-compatible computer system described by Stoddard and Loftus (1988).

Design and procedure. For each observer, 24 conditions were defined (four intensity levels and six exposure durations within each intensity level). The exposure-duration values within each intensity level were selected with the goals of (1) producing roughly equal performance ranges within each intensity level (which meant that durations within the lower intensity levels had to be suitably longer than corresponding durations within the higher intensity levels), and (2) maintaining performance (proportion of correctly recalled digits) within a range of roughly $0.1-0.9$.

Within each observer's intensity level, the six exposure durations were specified by two experimental parameters: base, the minimum duration, and factor, the amount by which each duration was multiplied to obtain the next higher duration. Table 2 provides these parameters for each of the 16 observer/intensity-level combinations.

Each observer participated in 24 blocks of 80 trials per block. Recall that stimuli were prepared as three 4-digit rows. On any given block, only one row (top, middle, or bottom) was the target to be reported. Also, stimulus intensity remained constant over a block. Intensity was changed on a given block by adjusting the luminance of the target-slide projector using the neutral-density filters. A uniform adapting field remained at a constant level (of $21.21 \mathrm{~cd} / \mathrm{m}^{2}$ ) at all times during an experimental session.

The sequence of events for a given 80-trial block was as follows. First, the observer ascertained that the filter configuration was correct for that block's intensity level (noting in the process what the stimulus intensity would be on that block). Next, a high, medium, or low tone $(2000,1000$, or $500 \mathrm{~Hz})$ signaled the observer that the top, middle, or bottom row would be the target row for that block (i.e., for the next 80 trials). Eight practice trials were then presented. The durations for these practice trials were selected randomly and without replacement. Next, 72 experimental trials were presented. The six durations were randomly intermingled over the 72 trials, with the restriction that each duration occurred 12 times. Stimulus presentation order was quasirandom. ${ }^{3}$

The row to be reported was changed systematically over blocks, so that it appeared in the top, middle, and bottom position in sequence; thus, each row served as target in 8 of the 24 blocks. As-

Table 2

Parameters for Generating Exposure Durations for Each Observer and Intensity Level

\begin{tabular}{ccccc}
\hline & \multicolumn{4}{c}{ Observer } \\
\cline { 3 - 5 } Intensity & C.A. & K.G. & G.L. & E.R. \\
\hline Level 1 & & & & \\
Base & 26 & 16 & 18 & 33 \\
Factor & 1.22 & 1.21 & 1.32 & 1.32 \\
Level 2 & & & & \\
Base & 18 & 13 & 10 & 14 \\
Factor & 1.23 & 1.18 & 1.40 & 1.32 \\
Level 3 & & & & \\
Base & 8 & 6 & 6 & 11 \\
Factor & 1.24 & 1.17 & 1.41 & 1.37 \\
Level 4 & & & & \\
Base & 6 & 5 & 3 & 6 \\
Factor & 1.23 & 1.17 & 1.38 & 1.32 \\
\hline
\end{tabular}

Note. Beginning with the lowest duration ("base" in $\mathrm{ms}$ ), each successive duration was computed by multiplying its predecessor by "factor." signment of durations to trials within a block was also changed over blocks, as was intensity. Each intensity level occurred once with each 4-block sequence. Although observers were not forced to participate in all 24 blocks at once, they were required to participate in 4-block modules, each of which incorporated all four intensity conditions. The 24 total blocks for each observer included two instances of each of the 12 intensity-level/to-bereported-row combinations.

As noted, a block consisted of 80 trials. The sequence of events within each trial was as follows. First, a 500 -ms tone warned the observer to look at a small fixation point that simultaneously appeared, superimposed over the adapting field, positioned such that it would be in the middle of the upcoming stimulus (i.e., between the second and third digits of the middle row). Warning-tone frequency was 2000,1000 , or $500 \mathrm{~Hz}$ and reminded the observer which row (top, middle, or bottom) was the target during the current block. Following the warning tone, the stimulus, superimposed on the adapting field, was presented for its appropriate duration. The observer typed in four responses after stimulus presentation, guessing on a digit if uncertain. After a response was made, feedback was given in the form of four 150-ms beeps. Each beep was $2000 \mathrm{~Hz}$ if the corresponding digit had been correctly reported and $500 \mathrm{~Hz}$ if the corresponding digit had not been correctly reported. This feedback was followed by a 300 -ms interval, after which the next trial began.

\section{Results}

Our basic performance measure, $p$, is the proportion of digits recalled in the correct position (corrected for the 0.10 chance rate).

Performance curves. We present our data in the form of performance curves, which are functions relating performance to exposure duration with different curves for different intensity levels. Past work using this paradigm (e.g., Busey \& Loftus, in press; Loftus, Busey, \& Senders, 1993; Loftus, Duncan, \& Gehrig, 1992; Shibuya \& Bundesen, 1988; Townsend, 1981) indicates that performance curves can be described almost perfectly by the equation

$$
p=\begin{array}{ll}
0 & \text { for } d \leq L \\
\mathrm{~A}\left[1.0-e^{-(d-L) / c}\right] & \text { for } d>L
\end{array}
$$

where $A, L$, and $c$ are free parameters. The interpretations of the parameter values are as follows. First, $L$ (mnemonic for "liftoff") is the performance curve's $d$-intercept (i.e., the minimum stimulus duration necessary for above-chance performance). Second, $c$ is the postliftoff duration (i.e., the duration exceeding $L$ ) required for performance to reach a criterion level of $p=A(1.0-1 / e)$. Finally, $A$ is asymptotic

\footnotetext{
${ }^{3}$ The 80 stimulus slides were fixed in the 80 slots of a carousel tray. We wanted to preclude observers' ability to memorize and make use of sequential, slide-to-slide information (e.g., we did not want middle row "7184" to always follow middle row "0072"). We accomplished this goal as follows. On each block, the 72 experimental stimuli were randomly divided into two 36-stimulus groups (Group A and Group B). The carousel circled twice within each block: on Pass 1, all Group A stimuli were shown, and on Pass 2, all Group B stimuli were shown. This scheme ensured that stimulus ordering differed unpredictably from one block to the next.
} 

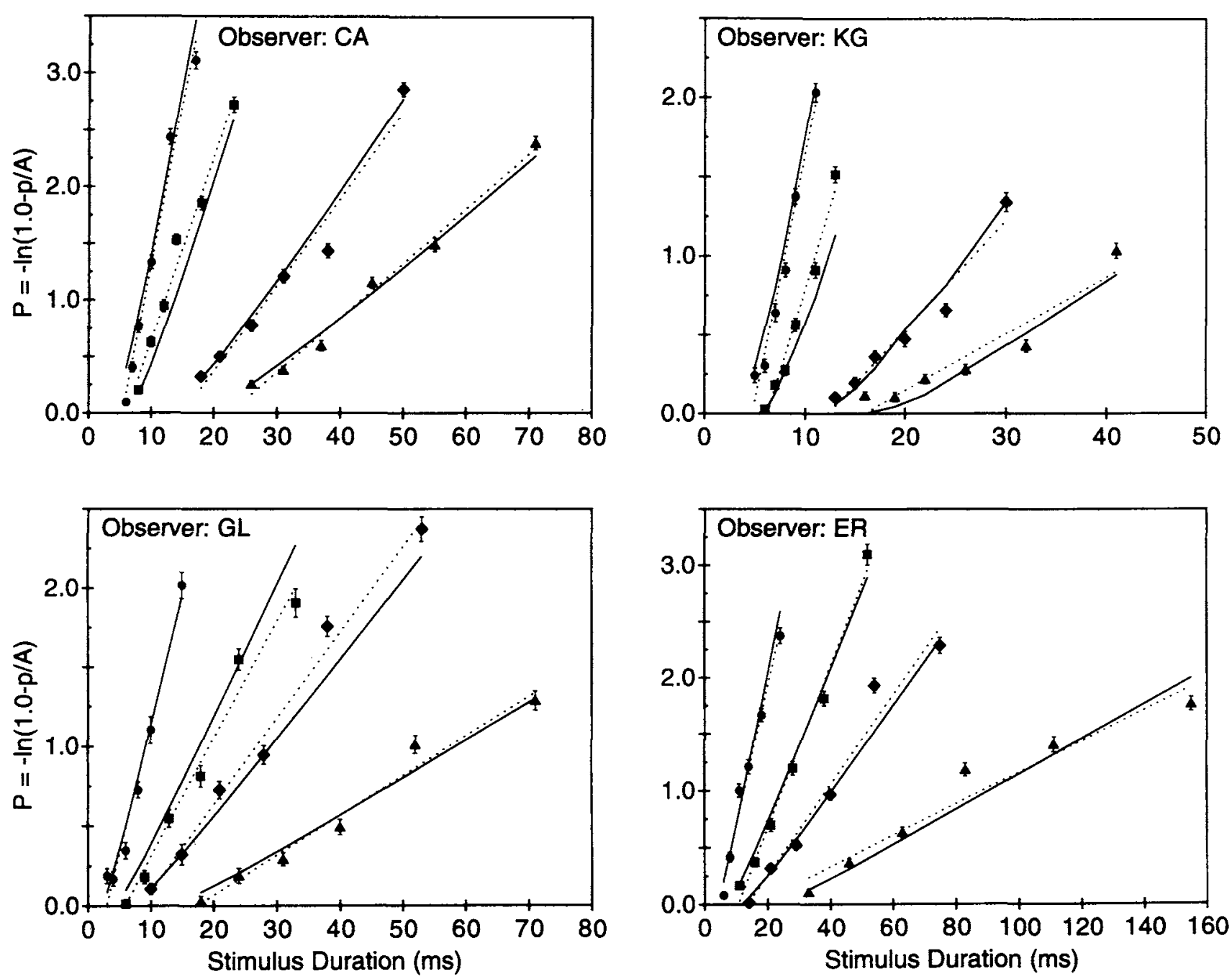

Figure 1. Experiment 1: Performance curves (recall performance measured in $P=-\ln (1.0-p)$ as functions of stimulus duration). Each panel shows data from one observer. The four curves within each panel are for the four intensity levels. The curve symbols represent data points (lowest intensity, triangles; next intensity, diamonds; next intensity, squares; and highest intensity, circles). Dashed lines are best linear fits, and solid lines are best fits from the theory described in the text.

performance. In the present paradigm, observers had the capability of reporting all four digits perfectly following sufficiently long exposure durations; thus, any $A$ values lower than 1.0 resulted from keypress errors, lack of vigilance, and so on. ${ }^{4}$ Accordingly, the value $A$ is uninteresting from the present perspective. We estimated $A$ for each observer, but we do not discuss it further. ${ }^{5}$

For simplicity of analysis and discourse, we define a new dependent variable, $P$, to be $P=-\ln (1.0-p / A)$, which, given Equation 2, implies

$$
P=\begin{array}{ll}
0 & \text { for } d \leq L \\
(l / c) d-L / c & \text { for } d>L .
\end{array}
$$

That is, if Equation 2 holds, then the performance measure $P$ is related linearly to duration with a slope of $1 / c$ and a $P$-intercept of $-L / C$. We assert that a performance curve's slope can be interpreted as the rate at which information is acquired from the stimulus: The greater the slope, the higher the information-acquisition rate. For the moment, we may consider this assertion to be "intuitively reasonable"; later, we see that it is implied by our theory.

Figure 1 shows the performance curves. Each panel represents the scores of one of the four observers; within each panel, the four curves represent the four intensity levels, with increasing intensities corresponding to leftward curves. The dashed lines through the data points are the best-fitting re-

\footnotetext{
${ }^{4}$ To ascertain that the lower-than- 1.0 asymptotic values were not perceptual effects, we carried out a control experiment in which stimuli were displayed as long as the observer wanted. After the observer signalled "enough," the stimuli were removed, and the observer responded. Performance was essentially $100 \%$.

5 The estimated asymptotes were 0.90 (C.A.), 1.00 (K.G.), 0.97 (G.L.), and 0.89 (E.R.).
} 
gression lines obtained using Equation 3. The solid lines are predictions from the theory that we describe below. The increasing performance-curve slopes with higher intensities indicate that with higher intensities, stimulus information is acquired at a higher rate. Table 3 provides the regression data $-C, L$, and Pearson $r^{2}$ values-for each observer and each intensity. It also provides root-meansquare errors $\left(\mathrm{rms}_{\mathrm{e}}\right)$ between the data points and predicted regression values.

Two aspects of these data are notable. First, the performance curves are well fit by linear functions. Of the 16 curves, one produces a Pearson $r^{2}$ of 0.90 , and the others all produce $r^{2} s$ of 0.94 or higher. Nine of the $16 r^{2} s$ are 0.98 or higher. This replicates past data using this paradigm (Busey \& Loftus, in press; Loftus, Busey, \& Senders, 1993; Loftus, Duncan, et al., 1992; Shibuya \& Bundesen, 1988). Second, the parameters $c$ and $L$ both decrease with increasing intensity. This means that with increasing intensity, (a) a smaller stimulus duration is required for performance to exceed chance (implied by decreasing $L$ ) and (b) once performance has exceeded chance, less additional stimulus duration is required to achieve any given criterion performance level (implied by decreasing $c$ ).

Are the performance curves fundamentally linear? Although there are deviations from linearity in the individual subject curves, they do not seem to be systematic. Figure 2 shows the mean performance curves (across observers) normalized for the different exposure durations. Again, dashed lines represent the best linear fits, and solid lines are theoretical predictions that are discussed in the next section. It is clear that the mean curves are highly linear; all $r^{2}$ values exceed 0.99 . Accordingly, we conclude that individual deviations from linearity are nonsystematic and that something

Table 3

Regression Fits for Each Observer and Each Intensity Level

\begin{tabular}{crrrr}
\hline & \multicolumn{4}{c}{ Observer } \\
\cline { 3 - 5 } Intensity & C.A. & K.G. & G.L. & E.R. \\
\hline Level 1 & & & & \\
$c$ & 20.69 & 27.70 & 40.19 & 72.46 \\
$L$ & 22.59 & 15.94 & 17.27 & 16.02 \\
$r^{2}$ & 0.99 & 0.90 & 0.97 & 0.94 \\
Level 2 & & & & \\
$c$ & 13.11 & 14.45 & 18.39 & 25.22 \\
$L$ & 15.16 & 12.29 & 8.42 & 13.48 \\
$r^{2}$ & 0.96 & 0.95 & 0.99 & 0.97 \\
Level 3 & & & & \\
$c$ & 6.12 & 4.74 & 13.51 & 13.98 \\
$L$ & 6.13 & 6.26 & 5.91 & 10.55 \\
$r^{2}$ & 0.98 & 0.98 & 0.98 & 0.99 \\
Level 4 & & & & \\
$c$ & 3.53 & 3.18 & 6.27 & 8.09 \\
$L$ & 5.38 & 4.75 & 2.91 & 4.39 \\
$r^{2}$ & 0.98 & 0.98 & 0.97 & 0.99 \\
& & & & \\
\hline rms $_{\mathbf{e}}$ (regressions) & 0.155 & 0.110 & 0.113 & 0.153 \\
\hline
\end{tabular}

Note. $c$ and $L=$ parameter values; $r^{2}=$ Pearson values; $\mathrm{rms}_{\mathrm{c}}=$ overall root-mean-square errors.

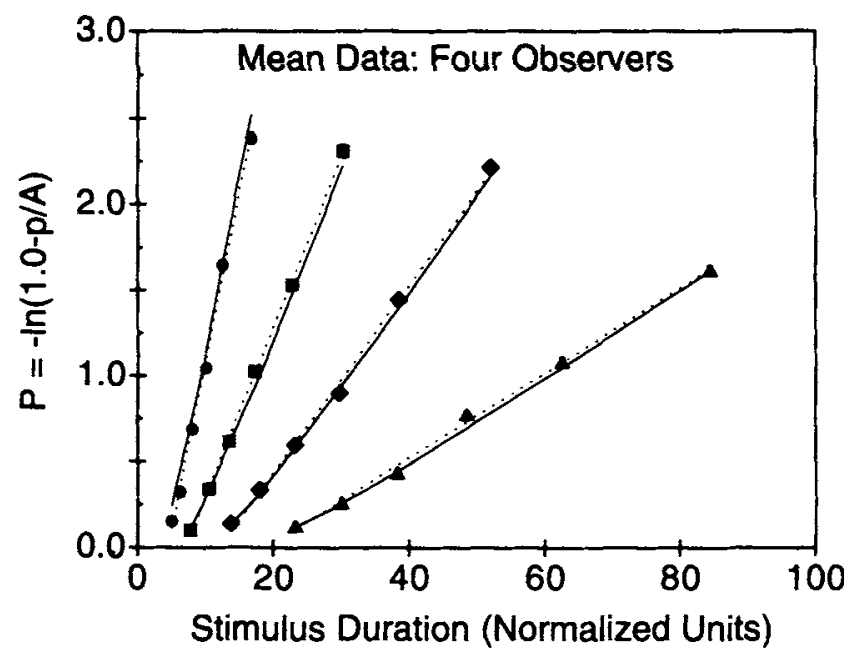

Figure 2. Experiment 1: Mean performance curves (recall performance as functions of duration, averaged across observers, normalized for different exposure durations). The four curves are for the four intensity levels. The curve symbols represent data points (lowest intensity, triangles; next intensity, diamonds; next intensity, squares; and highest intensity, circles). Dashed lines are best linear fits, and solid lines are best mean fits from the theory described in the text.

very close to linearity (Equation 3) accurately describes performance curves in this paradigm.

Intensity-duration trade-offs. We have shown that with higher intensity, performance increases faster with stimulus duration. We now consider the intensity-duration relations in more detail and in particular inquire whether they conform to Bloch's Law.

Our general logic is as follows. Consider some criterion performance level, $P_{c}$. Bloch's Law asserts that the product of duration, $d$, and intensity, $\phi$, required to achieve $P_{c}$ is constant; in other words,

$$
\phi d=k_{1},
$$

where $k_{1}$ is a constant, or

$$
\frac{1}{d}=k_{2} \phi,
$$

where $k_{2}=1 / k_{1}$ is also a constant. Thus, the prediction is that across intensity levels, the reciprocal of $d$ will be linearly related to stimulus intensity with an intercept of zero.

What should we use as $\boldsymbol{P}_{c}$, the criterion performance level? As indicated by the shape of our performance curves, increasing stimulus duration leads to two successive achievements. First, at duration $L$, performance rises above chance. Second, with additional duration beyond $L$, performance rises linearly. The Bloch's Law prediction (Equation 4) can be tested for both these effects. With the first performance criterion being the rise from chance that occurs at duration $L \mathrm{~ms}$, the prediction becomes

$$
\frac{1}{L}=k_{L} \phi
$$

where $k_{L}$ is a constant. 
With the second performance criterion being the achievement of $P=1.0$, which occurs at duration $c$ following liftoff, the prediction becomes

$$
\frac{1}{c}=k_{c} \phi
$$

where $k_{c}$ is a constant.

One important note is in order here. The criterion performance level of $P=1.0$ used as a basis for determining the duration $c$ is, of course, arbitrary. However, because the performance curves are linear beginning at duration $L$, using a different criterion performance level (call it $P^{\prime}$ ) would simply entail a rescaling of the original $c$ values by a factor of $P^{\prime}$ across intensity levels. Equation 6 would still hold, although the constant of proportionality ( $k_{c}$ in Equation 6) would be different.

Equations 5 and 6 thus make analogous predictions for each of the performance curve parameters, $L$ and $c$ : The parameter's reciprocal should be proportional to intensity, $\phi$. For the record, we can also test an analogous "standard" Bloch's Law prediction: The total duration $(L+c)$ required to achieve any criterion performance level trades off with intensity. This prediction is that

$$
\frac{1}{L+c}=k_{L+c} \phi
$$

where $k_{L+c}$ is a constant. We must, however, regard the prediction of Equation 7 with some wariness, because unless the ratio $L / c$ is constant across intensity levels, Equation 7's validity will depend on the particular performance level that is chosen. There is no a priori reason to expect any particular relation between $L$ and $c$.

Recall that Observers C.A. and K.G. had one intensity range, while Observers G.L. and E.R. had a different intensity range. Figure 3 shows $1 / L, 1 / c$, and $1 /(L+c)$ as functions of intensity averaged over C.A. and K.G. (top panel) and over G.L. and E.R. (bottom panel). As in Figures 1 and 2, the dashed lines represent the best linear fit, and the solid lines represent theoretical predictions discussed later. It is clear that the predictions embodied in Equations 5-7 hold quite well: As indicated in the figure legends, the curves are quite linear, and the intercepts are quite close to zero.

\section{Discussion}

The purpose of Experiment 1 was to determine the relations between stimulus duration and stimulus intensity in a digit-recall task. Several findings emerged. First, in accord with other studies using this paradigm (e.g., Loftus, Duncan, et al., 1992), performance as a function of stimulus duration was described well by a linear function (or by an exponential approach to an asymptote if proportion correct is the dependent variable). This finding, demonstrated in Figures 1 and 2, is interesting in and of itself, and is discussed in detail by Loftus, Busey, and Senders (1993). For present purposes, however, we regard this performance curve simplicity as a convenient tool for carrying out other analyses.
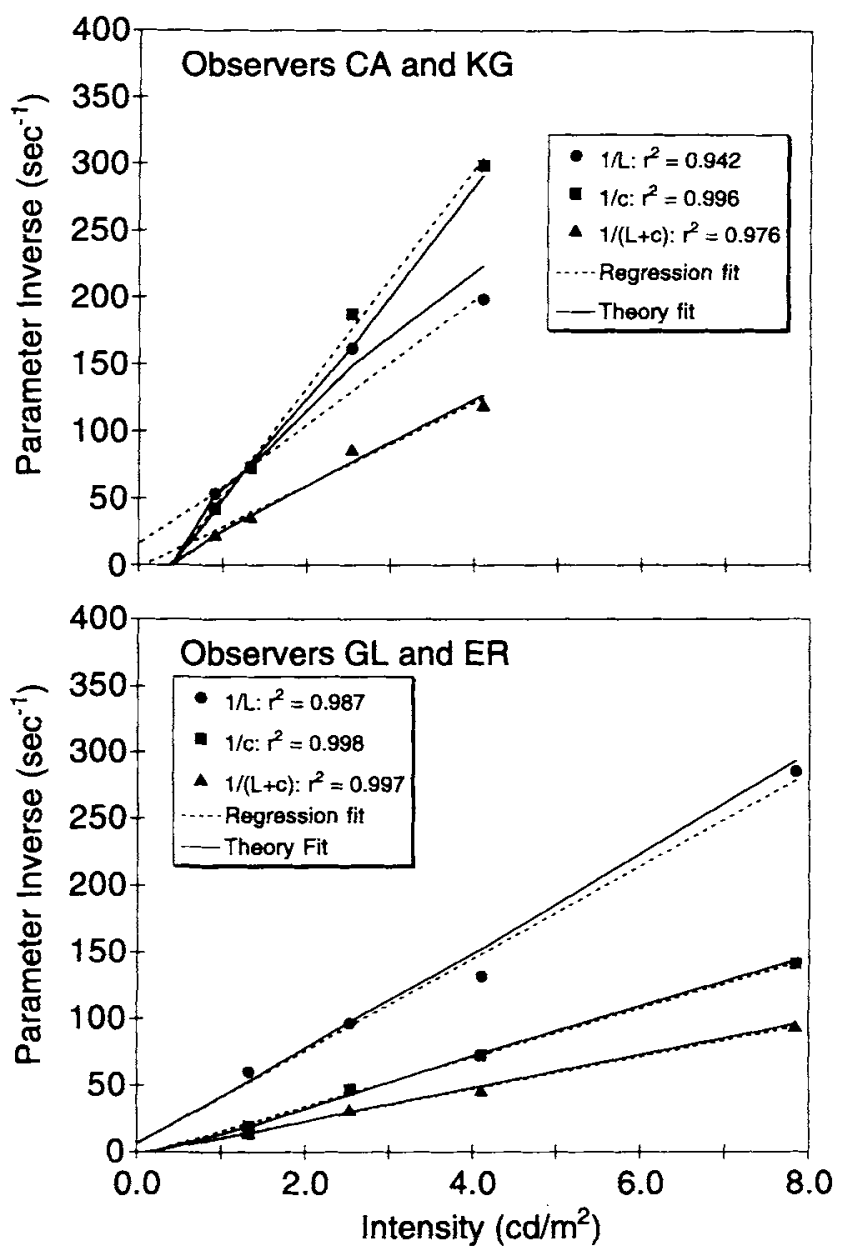

Figure 3. Experiment 1: Each panel shows $1 / L, 1 / c$, and $1 /(L+c)$ as functions of stimulus intensity averaged over two observers. Dashed lines are best linear fits, and solid lines are best fits from the theory described in the text.

In particular, the nature of the performance curves facilitated a detailed investigation of the relation between duration and intensity. As indicated in Figure 3, we discovered that Bloch's Law held quite well in terms of two fundamental durations and associated performance criteria. First, intensity traded off with $L$, the initial duration required for performance to exceed chance. Second, intensity traded off with the additional duration required to rise from chance to any criterion above-chance performance level. At the risk of redundancy, we reemphasize that this latter duration is equal to the regression parameter $c$ if a criterion performance level of $P=1.0$ is used. However, the linearity of the performance curves implies that the trade-off between intensity and postliftoff duration will hold independent of the particular above-chance criterion performance level that is chosen.

In addition to the trade-offs between intensity on the one hand and the durations $L$ and $c$ on the other hand, we discovered that intensity also trades off quite well with the total duration required to achieve above-chance performance. 
This observation indicates that the relation between the durations $L$ and $c$ is not arbitrary; rather, it is such that the ratio $c / L$ is independent of intensity level.

In short, we have discovered strong regularities in the duration-intensity relation underlying performance in a digit-recall task. These regularities suggest that performance in this task can be described by a relatively simple theory of perception, memory, and immediate recall. We now describe such a theory.

\section{A Linear-Filter/Information-Acquisition Theory}

The theory we describe in this section is a concatenation of two models that have been used in the past to describe two different domains: low-level visual processes and higher level cognitive processes.

\section{Overview}

We refer to the first component as the sensory-response model. We assume, in particular, that the initial stages of the visual system act as a linear low-pass temporal filter that operates on a physical stimulus, to produce what we term a sensory-response function, designated $a(t)$. This function relates the magnitude of some form of neural activity associated with stimulus presence to time $t$ since stimulus onset. ${ }^{6}$ This kind of temporal-filter model has been used to account for a variety of low-level visual phenomena such as flicker detection and time-intensity relations in simple detection tasks (see Watson, 1986, for an overview); however, linearfilter models have been used only sporadically to account for higher level cognitive phenomena (e.g., Dixon \& Di Lollo, in press; Groner, Bischof, \& Di Lollo, 1988).

The second component of the theory, termed the acquisition-rate model, has been described by Loftus and his colleagues to account for relatively high level pictureprocessing tasks ${ }^{7}$ and builds on several earlier models. ${ }^{8}$ The acquisition-rate model begins with the assumption that there is some sensory-response function, $a(t)$, that rises following stimulus onset and decays following stimulus offset. ${ }^{9}$ The model further assumes the following. First, information is acquired from a stimulus and placed into a more permanent memory where it can be used as the basis for either an immediate response or further cognitive processing. Second, such information acquisition occurs at a rate, $r(t)$, that is, among other things, proportional to $a(t)$. Third, any measure of subsequent memory performance is monotonically related to the amount of acquired information.

We will show that this model makes an important prediction: that memory performance is monotonically related to total area under the $a(t)$ function, which we designate $A(\infty)$. Thus, any two stimuli that engender the same area (e.g., a short, high-intensity stimulus and a longer, lower intensity stimulus) must lead to identical performance. As we shall see, it is this property of the model that allows it to account for the intensity-duration relation that we observed in Experiment 1.

The acquisition-rate model and the sensory-response model are described in detail in the following sections. We describe the acquisition-rate model first as this component presupposes some sensory-response function.

\section{Acquisition-Rate Model}

The logic of the acquisition-rate model is as follows. First, the physical stimulus presentation engenders a sensory response. The sensory response forms the basis for acquisition of stimulus information, which in turn leads to the memory representation on which subsequent memory performance depends. More precisely,

1. A physical stimulus is characterized as a temporalinput function, $f(t)$, relating stimulus intensity to the time $t$ since stimulus onset. The left panels of Figure 4 illustrate six $f(t)$ functions: These are square-wave functions of the sort used in most perceptual experiments, including the present Experiment 1 . Because they are square-wave functions, the term intensity can be informally used to describe the function's maximum intensity. Intensity is 1.0 in the first three panels (page 40), and 2.0 in the last three panels (page 41).

2 . The stimulus input function engenders the sensoryresponse function $a(t)$. The $a(t)$ functions resulting from the Figure 4 stimuli are shown in the right panels of Figure 4. The vertical lines represent stimulus offset. ${ }^{10}$ Generally speaking, $a(t)$ lags behind and is temporally blurred in relation to $f(t)$. Below we describe both the mathematical origin of these functions and the meaning of the horizontal lines just above the abscissas.

3. The subject's task is construed as acquiring information from the stimulus and transferring it to more permanent storage. At time $t$ following stimulus onset, some proportion $[I(t)]$ of all stimulus information has been acquired. Information is acquired at an instantaneous rate, $r(t)$, which is the derivative over time of acquired information, $d[I(t)] / d t$. The

\footnotetext{
${ }^{6}$ The designation of the sensory response function as $a(t)$ is historical. In previous formulations of the model, the $a(t)$ function has been termed "proportion of available information" and was constrained to vary between 0.0 and 1.0 . In the present formulation, the conceptual definition has been broadened, and the range constraint has been dropped.

${ }^{7}$ See, for example, Loftus and Hogden (1988) and Loftus, Hanna, and Lester (1988) for general descriptions. The model is applied to temporal-integration tasks by Loftus and Hanna (1989) and Loftus and Irwin (1993). It is applied by Busey and Loftus (in press) and Loftus, Busey, and Senders (1993) to the sort of digitrecall task used in the present Experiment 1. It is applied to partial report by Loftus and Busey (1992) and Loftus and Irwin (1993). Di Lollo and Dixon (1992) presented an opposing viewpoint.

${ }^{8}$ Notably, Bundesen (1990), Kowler and Sperling (1980), Loftus and Kallman (1979), Massaro (1970), Rumelhart (1969), and Townsend (1981).

${ }^{9}$ In previous formulations of the model, this $a(t)$ function was defined somewhat arbitrarily-that is, on the basis of intuitionrather than generated on the basis of fundamental principles.

10 The portion of $a(t)$ that follows stimulus offset can be identified with the iconic image.
} 

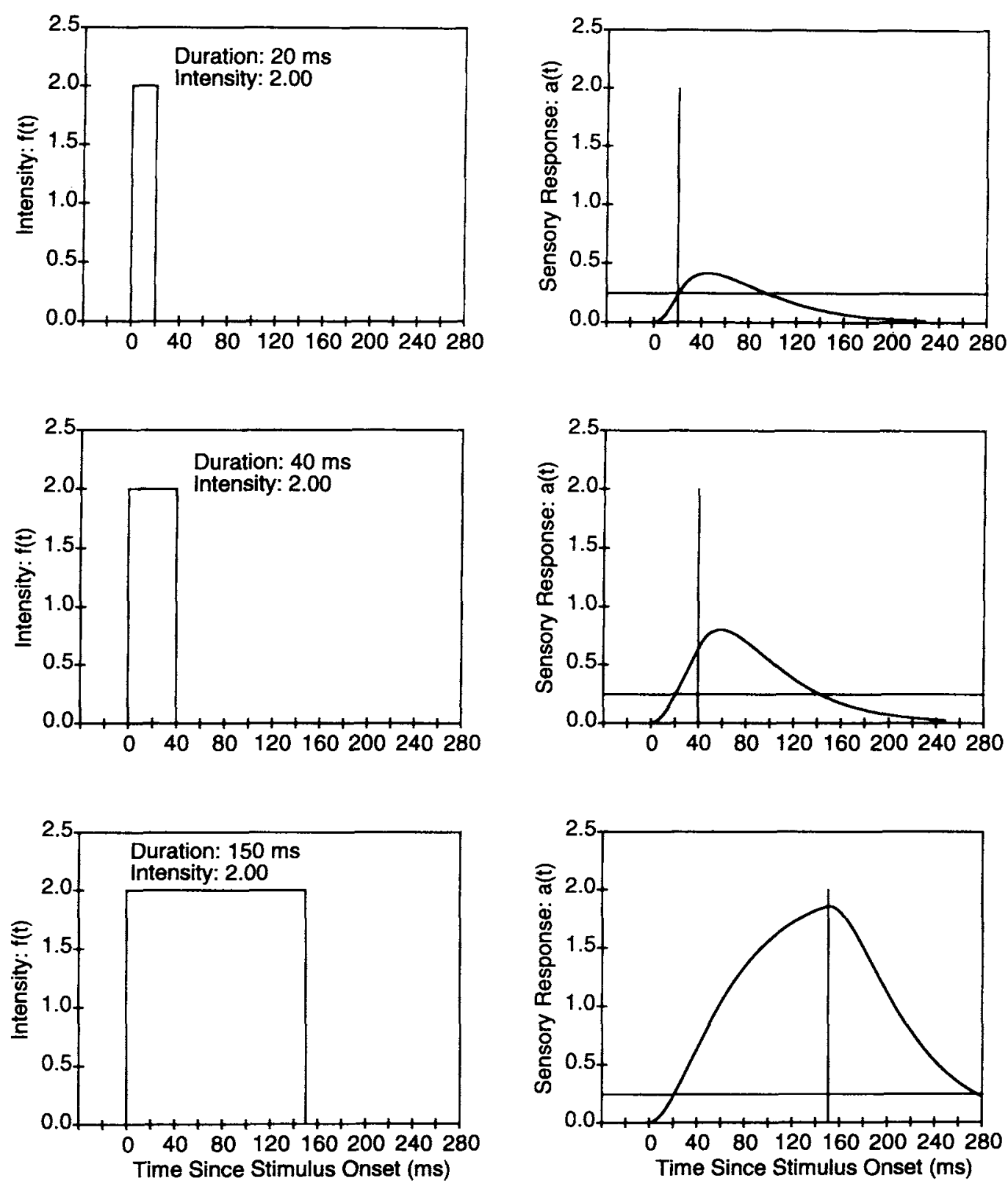

Figure 4. Stimulus input functions ( $f[t]$; left panels) and the resulting sensory-response functions ( $a[t]$; right panels). In the $a(t)$ functions, the vertical line represents the time of stimulus offset, and the horizontal lines represent sensory-threshold values.

value of $r(t)$ is the product of two entities: first, $a(t)$, and second, some function $h[I(t)]$ of already acquired information. The only constraints on $h[I(t)]$ are that it is positive, finite, monotonically decreasing, and zero when $I(t) \leq 1.0$. Thus, new information is acquired at a rate that is proportional to the sensory response and inversely related to amount of already acquired information. ${ }^{11}$ Note that given the constraints on $r(t), I(t)$ cannot exceed 1.0.

4. Performance, $P$, is a monotonic function, $m[I(\infty)]$, of the total acquired information, $I(\infty)$.

Appendix A shows that given the logic thus far, a powerful prediction ensues: Performance $p$ is a monotonic function of the total area under the $a(t)$ function. We term this total area $A(\infty)$. Others (e.g., Nisly \& Wasserman, 1989; see their Figure 2) have informally proposed that the area under some sensory-response function might be suitable as a theoretical

${ }^{11}$ These assumptions imply that $r(t)$ generally declines following stimulus onset as a result of the decreasing $h[I(t)]$ component. Following stimulus offset, $r(t)$ begins to decline more precipitously as a result of the additional decrease in the $a(t)$ component. Clark and Hogben (1991) offered a visual-processing model in which system output is defined by fiat to have this form. 

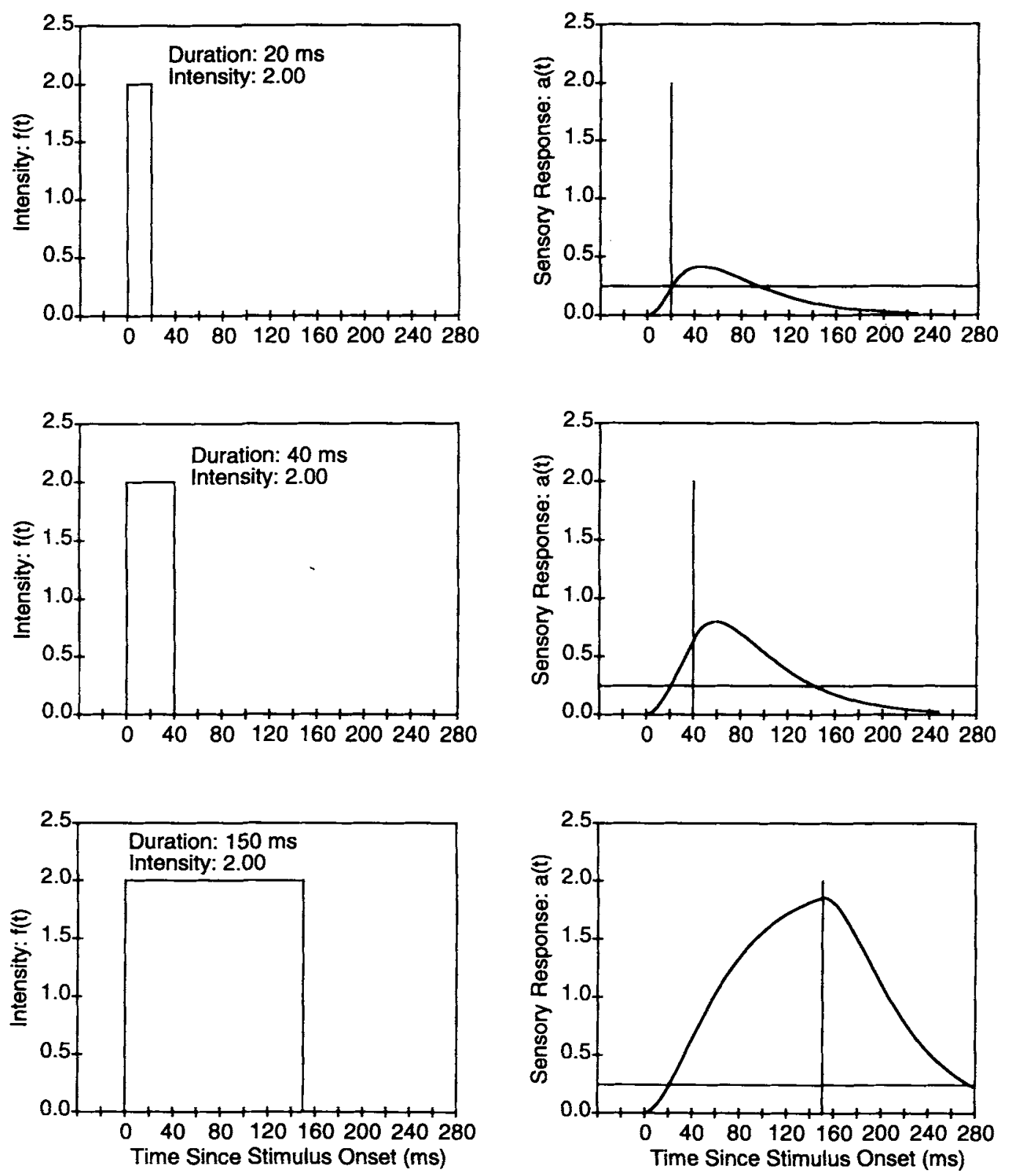

Figure 4, continued.

basis of stimulus identification. The reasoning that we have just provided establishes a formal basis for this proposition.

\section{Sensory-Response Model}

Where does the presumed sensory-response function $a(t)$ come from? Loftus, Duncan, et al. (1992) assumed a semiarbitrary $a(t)$ function but pointed out that it was deficient in that it did not derive from basic principles. Loftus et al. did, however, sketch a means by which the function could be derived from more basic principles based on the proposition, alluded to earlier, that a linear low-pass temporal filter operates on the stimulus input function to generate the sensoryresponse function.
Impulse and impulse-response function. The linearfilter-based model begins with the assumption that an input consisting of an instantaneous impulse $e^{12}$ engenders what is termed the impulse-response function. The impulse-response function is often assumed to be a gamma function of the form

$$
g(t)=\frac{(t / \tau)^{n-1} e^{-t / \tau}}{\tau(n-1) !},
$$

where $n$ and $\tau$ are free parameters: $n$ is a positive integer, and $\tau$ is a positive real number (cf. Watson, 1986). The impulse-

\footnotetext{
${ }^{12}$ More precisely, an impulse is defined as a stimulus of infinitesimal duration, infinite intensity, and unit area.
} 
response function of Equation 8 is illustrated in Figure 5. As noted in Figure 5 the total area under the impulse-response function is 1.0. The gamma function is rooted in physical reality, in that it describes the response of a system of $n$ independent stages where the input to Stage 1 is the impulse, the input to each subsequent stage is the response of the previous stage, and the response of each stage decays exponentially with decay constant $\tau$.

Linear responses to arbitrary input function. The model next assumes that any input function may be viewed as a series of impulses, scaled by intensity, and that the resulting sensory-response $[a(t)]$ function is the sum of the resulting linearly scaled impulse response functions. More precisely, given input function $f(t)$ and impulse-response function $g(t)$, the resulting sensory-response function $a(t)$ is the convolution of $f(t)$ and $g(t)$.

Sensory response to square-wave input. In Experiment 1 , we used square-wave displays as shown in the left panels of Figure 4: To display a stimulus, the projector shutter opened, essentially instantaneously, remained open for some duration $d$, and then closed, again essentially instantaneously. ${ }^{13}$ This makes computation of the $f(t)$ and $g(t)$ convolution quite simple. For a $d$-ms square-wave function whose maximum intensity is $\phi$, the resulting $a(t)$ function is

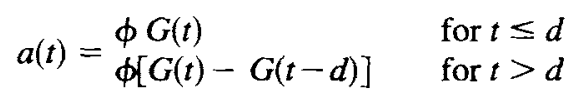

where $G(x)$ is the integral from zero to $x$ of $g(x) d x$. The $a(t)$ functions shown in the right panels of Figure 4 were generated from Equations 8 and 9.

\section{Accounting for Bloch's Law}

The theory that we have so far described is quite simple. In summary, an $a(t)$ function is generated by a linear filter that operates on the stimulus input function. Information is acquired at a rate $r(t)=a(t) h[I(t)]$, and performance $P$ is

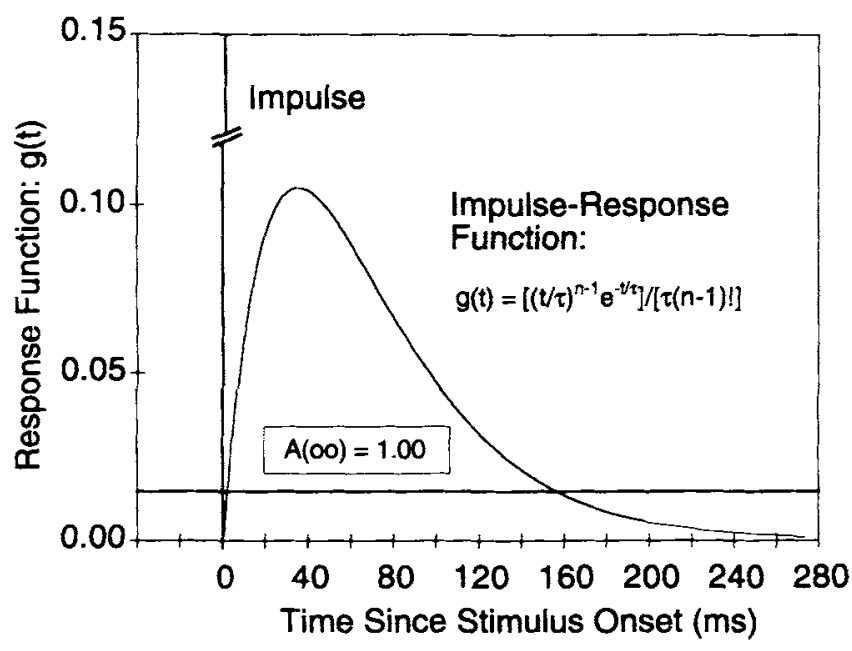

Figure 5. An impulse and the resulting impulse-response function. a monotonic function $m$ of $I(\infty)$, the total information acquired from the stimulus. As we have noted, this theory implies performance $P$ to be a monotonic function of $A(\infty)$, the total area under the sensory-response function, $a(t)$. Because $a(t)$ is generated by a linear process operating on $f(t)$, the total area under $a(t), A(\infty)$, must be proportional to the total area under $f(t), F(\infty)$, the constant of proportionality being $\phi$, the intensity.

It is easy to see that the theory as described so far implies something close to our Bloch's Law finding. The $f(t)$ function is a rectangle whose width is duration and whose height is intensity. Because performance is monotonically related to $A(\infty)$, it is also monotonically related to $F(\infty)$, which is the rectangle's area. This implies a perfect duration/intensity trade-off: If duration is multiplied by some factor, intensity must be divided by the same factor to maintain equal area.

\section{Accounting for Experiment I Data}

So far, however, the theory does not account for either the linear performance curves or the nonzero liftoff values that we observed in Experiment 1. To remedy these deficits, we require two modifications to the theory. First, the linear performance curves result if the presumed monotonic functions, $h[I(t)]$ and $m[I(\infty)]$, are strengthened in suitable ways. Second, the nonzero liftoff values result with one additional assumption about the nature of the information-acquisition rate, $r(t)$.

Linear performance curves: Strengthening the $h[I(t)]$ and $m[I(\infty)]$ functions. In Experiment 1 we observed linear performance curves. The theory predicts such linearity if the functions $h[I(t)]$ and $m[I(\infty)]$ presently assumed to be only monotonic are strengthened such that

$$
h(I)=\frac{1.0-\mathrm{I}(t)}{c},
$$

where $c$ is a free parameter, and

$$
m[I(\infty)]=I(\infty)
$$

These strengthened assumptions are both reasonable. Equation 10 asserts that the information-acquisition rate is proportional to the remaining to-be-acquired information at any given time. This relation, implied as it is by an ecologically common Poisson process, describes many physical processes, such as radioactive decay. Equation 11 simply asserts that proportion correct is equal to the proportion of acquired information. Appendix B shows that with these strengthened assumptions, performance curves are described by the equation

$$
P=\frac{d}{c}
$$

where the parameter $c$ is proportional to $1 / \phi$, the inverse of stimulus intensity.

\footnotetext{
${ }^{13}$ In fact, it took approximately $2 \mathrm{~ms}$ both for the shutters to completely open and for them to completely close.
} 
Equation 12 almost, but not quite, describes the observed performance curves. According to Equation 12, all performance curves pass through the origin. However, the observed performance curves do not pass through the origin. Instead, as we have seen (Figures 1 and 2), the observed performance curves intersect the duration axis at some positive value $L$ that increases with decreasing intensity. The theory is, accordingly, still deficient.

Nonzero liftoffs: The threshold assumption. This deficiency can be remedied by inclusion of one additional assumption: that information acquisition does not begin until the sensory-response function exceeds some threshold. This threshold is represented by the horizontal lines just above the abscissas in the right-hand panels of Figure 4 . We refer to this sensory threshold value as $a_{t}$.

The simplest way of incorporating such a threshold within the theory is to assume that the information-acquisition rate, $r(t)$, is proportional not to $a(t)$ but to the magnitude by which $a(t)$ exceeds $a_{t}$. It is easy to show that the theory then predicts performance to depend on the total area under the $a(t)$ function above threshold (see Appendix C). We refer to this value as $A_{t}(\infty)$. The performance-curve equation then becomes

$$
P=\frac{A_{t}(\infty)}{c}
$$

where $c$ is approximately proportional to $1 / \phi$. Note that $A_{t}(\infty)$ is zero for durations less than some threshold duration. For any given intensity level, the threshold duration is the longest duration such that $a(t)$ never exceeds the threshold $a_{r}$. These assertions are illustrated by the $a(t)$ functions in Figure 4 (right panels). Here, the horizontal lines represent threshold values. Note first that when intensity is 1.0 , the $d=20 \mathrm{~ms}$ $a(t)$ function never achieves threshold; accordingly, $A_{t}(\infty)$ - and performance- - would be zero for all values of 20 $\mathrm{ms}$ or less. When intensity is 2.0 , however, the $d=20 \mathrm{~ms}$ curve does exceed threshold; accordingly, $A_{t}(\infty)$ - and performance-would exceed zero. This threshold assumption thereby accounts qualitatively for two observed aspects of the data: first, that the critical liftoff stimulus duration generally exceeds zero and, second, that this liftoff value decreases with increasing intensity levels (see Figure 3, top panel). This revised theory no longer predicts exactly linear performance curves. As we shall see, however, it predicts performance curves that are almost linear.

\section{Theoretical Fit}

In summary, the theory has five free parameters: the asymptote, A; the two impulse-response function parameters, $n$ and $\tau$; the sensory-response threshold, $a_{t}$; and a scaling parameter, $c$. As noted earlier, the asymptote for each observer was already estimated in the process of producing the best linear performance curves and is not considered further here. In what follows, we consider only the parameters, $n, \tau$, $\mathrm{c}$, and $a_{r}$.

We found the best fitting values of these four parameters for each of the four observers with a grid search procedure. The goodness of fit was remarkably impervious to the value
Table 4

Model Fits for Each Observer

\begin{tabular}{|c|c|c|c|c|}
\hline \multirow{2}{*}{ Parameters } & \multicolumn{4}{|c|}{ Observer } \\
\hline & C.A. & K.G. & G.R.L. & E.R. \\
\hline \multicolumn{5}{|c|}{$n=2$} \\
\hline$\tau(\mathrm{ms})$ & 3.3 & 2.7 & 4.4 & 3.6 \\
\hline$c(\mathrm{~ms})$ & 12 & 10 & 44 & 52 \\
\hline$a_{t}\left(\mathrm{~cd} / \mathrm{m}^{2}\right)$ & 0.32 & 0.37 & 0.23 & 0.48 \\
\hline $\mathrm{rms}_{\mathrm{e}}($ model) & 0.210 & 0.145 & 0.154 & 0.171 \\
\hline \multicolumn{5}{|c|}{$n=10$} \\
\hline$\tau(\mathrm{ms})$ & 5.7 & 3.9 & 8.1 & 6.7 \\
\hline$c$ (ms) & 12 & 10 & 45 & 52 \\
\hline$a^{t}\left(\mathrm{~cd} / \mathrm{m}^{2}\right)$ & 0.35 & 0.48 & 0.25 & 0.52 \\
\hline $\mathrm{rms}_{\mathfrak{e}}($ model $)$ & 0.214 & 0.148 & 0.151 & 0.165 \\
\hline
\end{tabular}

Note. Within each $n$ value, first three rows show best-fitting values for the other three parameters, and the bottom row shows the root-mean-square error $\left(\mathrm{rms}_{\mathrm{e}}\right)$.

of $n$. This is demonstrated in Table 4, which shows the best fitting values of $\tau, c$, and $a_{t}$, along with the $\mathrm{rms}_{\mathrm{e}}$ when $n=$ 2 (top) and when $n=10$ (bottom). The $\mathrm{rms}_{\mathrm{e}}$ depends only very slightly on the value of $n$. The value of $c$ depends not at all on $n$, the value of $a_{t}$ depends slightly on $n$, and the value of $\tau$ depends quite a bit on $n$.

The threshold $\left(a_{t}\right)$ values are in intensity units (candelas per square meter). The rationale underlying this assertion is as follows. As the stimulus remains on indefinitely, $a(t)$ will asymptote at a value equal to the stimulus intensity level. Accordingly, the threshold can be interpreted as the maximum stimulus intensity level at which no information would be acquired even if the stimulus were of infinite duration.

Predicted performance curves. The predicted performance levels are shown as the solid lines in Figure 1 (individual data) and Figure 2 (mean data across the four observers). The predicted best fit $P$ values are essentially identical for any value of $n$ from 2 to 10 ; for the record, the predictions shown are based on $n=10$ (an $n$ value that often emerges when the linear-filter model is used to account for low-level sensory data; see Watson, 1986). The predicted mean performance curves (Figure 2) were generated by simply averaging the individual predicted curves, normalizing for different observers' different exposure durations (as was done to produce the mean data). It is obvious that the mean predicted curves correspond closely to the data and are virtually indistinguishable from their linear counterparts.

Predicted $1 / L, 1 / c$, and $1 /(L+c)$ curves. The predicted $1 / L, 1 / c$, and $1 /(L+C)$ curves are shown as the solid lines in Figure 3. Predicted values were obtained by finding the best linear fits ( $L$ and $c$ values) to the predicted performance curves for each observer and then averaging the resulting $1 / c$ and $1 / L$ values separately for C.A. and K.G. and for G.L. and E.R. (as was done to produce the data). The predicted intensity-axis intercepts were the estimated threshold values: If intensity is at (or below) the threshold value, no information is ever acquired; hence both $L$ and $c$ would be infinite, and their reciprocals would be zero.

Recall the Bloch's Law predictions embodied in Equations 
4 and 5: $1 / L$ and $1 / c$ are proportional to intensity. The theory makes something very close to this prediction; however, at small intensities, the predicted curves bow downward, and intercept the intensity axis at small positive values. These departures result from the theory's nonlinearity embodied in the sensory threshold.

We have argued that the most fundamental Bloch's Law predictions are those involving the trade-offs between intensity and the durations $L$ and $c$. Other research designed to investigate Bloch's Law has typically examined the trade-off between intensity and total duration. In Experiment 1, there turned out to be an almost-perfect trade-off between intensity and total duration $(L+c)$; as is evident in Figure 3, this tradeoff is predicted by our theory.

\section{Experiment 2: Additional Test of the Theory}

Experiment 2 was designed to test a straightforward implication of the theory's threshold assumption. To understand this prediction, consider for a moment the theory without the threshold assumption. Without a threshold, the theory would imply memory performance to depend on $A(\infty)$, the total area under the sensory-response function. $A(\infty)$ is, in turn, equal to $F(\infty)$, the total area under the stimulus input function.

Suppose now that we generate a stimulus of intensity, $\phi$, whose total duration is $d \mathrm{~ms}$. We create two conditions. In the first condition, the stimulus is simply presented as a square-wave function for the $d \mathrm{~ms}$. In the second condition, the $d \mathrm{~ms}$ is divided into two separate square-wave presentations of durations $d_{1} \mathrm{~ms}$, and $d_{2}=\left(d-d_{1}\right) \mathrm{ms}$, which are displayed successively, separated by some temporal gap. We refer to these conditions as the no-gap and the gap conditions, respectively. For instance, a no-gap condition might consist of a single $40-\mathrm{ms}$ stimulus, while the corresponding gap condition might consist of a 20 -ms stimulus, followed by a 250 -ms blank period, followed by the stimulus for another $20 \mathrm{~ms}$.

Figure 6 shows the $f(t)$ and $a(t)$ functions resulting from these two conditions. The two $a(t)$ functions are quite different from one another. However, because the system is linear, the total areas $A(\infty)$ must be the same; in the example, they both equal 40 . In the absence of a threshold, therefore, the two conditions are predicted by the theory to yield equal performance.

However, if a threshold is introduced, the no-gap condition is predicted to yield a higher $A_{t}(\infty)$ and hence higher performance than the gap condition. This mathematical truth may or may not be intuitively obvious, but the criterion has been selected in the Figure 6 example to illustrate it as forcefully as possible. In the gap condition, $a(t)$ never exceeds the threshold; hence $A_{t}(\infty)$ and performance are both zero. In the no-gap condition, however, $a(t)$ does exceed threshold; hence $A_{t}(\infty)$ and performance are both above zero. More generally, the prediction is that assuming a threshold, performance will be worse in the gap condition than in the no-gap condition.

\section{Method}

Experiment 2 was actually a collection of miniexperiments using the same stimuli and display procedures as Experiment 1 . In all miniexperiments, there was a gap and a no-gap condition. We used various combinations of $d_{1}, d_{2}$, and gap duration. With one exception, only a single intensity was used: $\phi=1.372 \mathrm{~cd} / \mathrm{m}^{2}$. Observers included G.L. along with graduate and undergraduate students working in the laboratory.
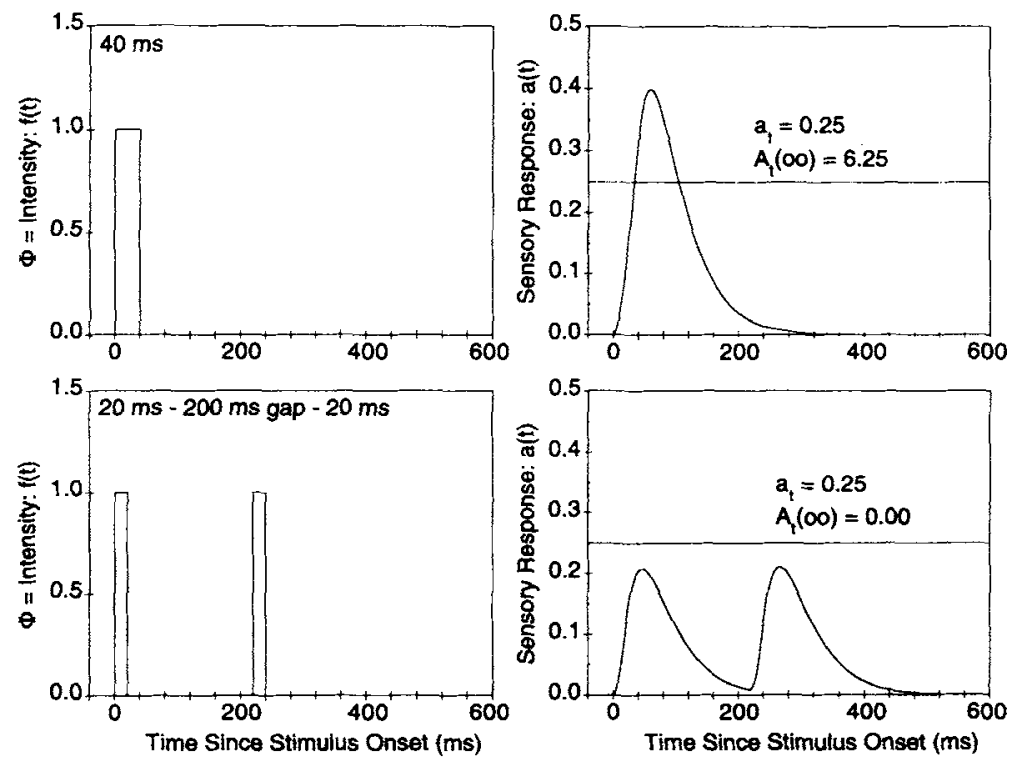

Figure 6. Experiment 2: Stimulus input functions $[f(t)$; left panels] and the resulting sensoryresponse functions $[a(t)$; right panels] for the no-gap and gap conditions. 


\section{Results and Discussion}

The results were clear-cut: Performance in the gap condition was invariably inferior to performance in the no-gap condition. This was true for all miniexperiments. Figure 7 illustrates these results from two of the miniexperiments. The theory's prediction is thus confirmed.

\section{General Discussion}

We first summarize what we have shown thus far. We then discuss two additional issues: first, the relations among Bloch's Law, the results of Experiment 1, and our theory; and second, the relation between initial phenomenological appearance of some visual stimulus and information acquired from that stimulus.

\section{Summary}

In Experiment 1, we demonstrated several strong regularities in the relations between stimulus intensity and stimulus duration in a digit-recall task: The product of stimulus
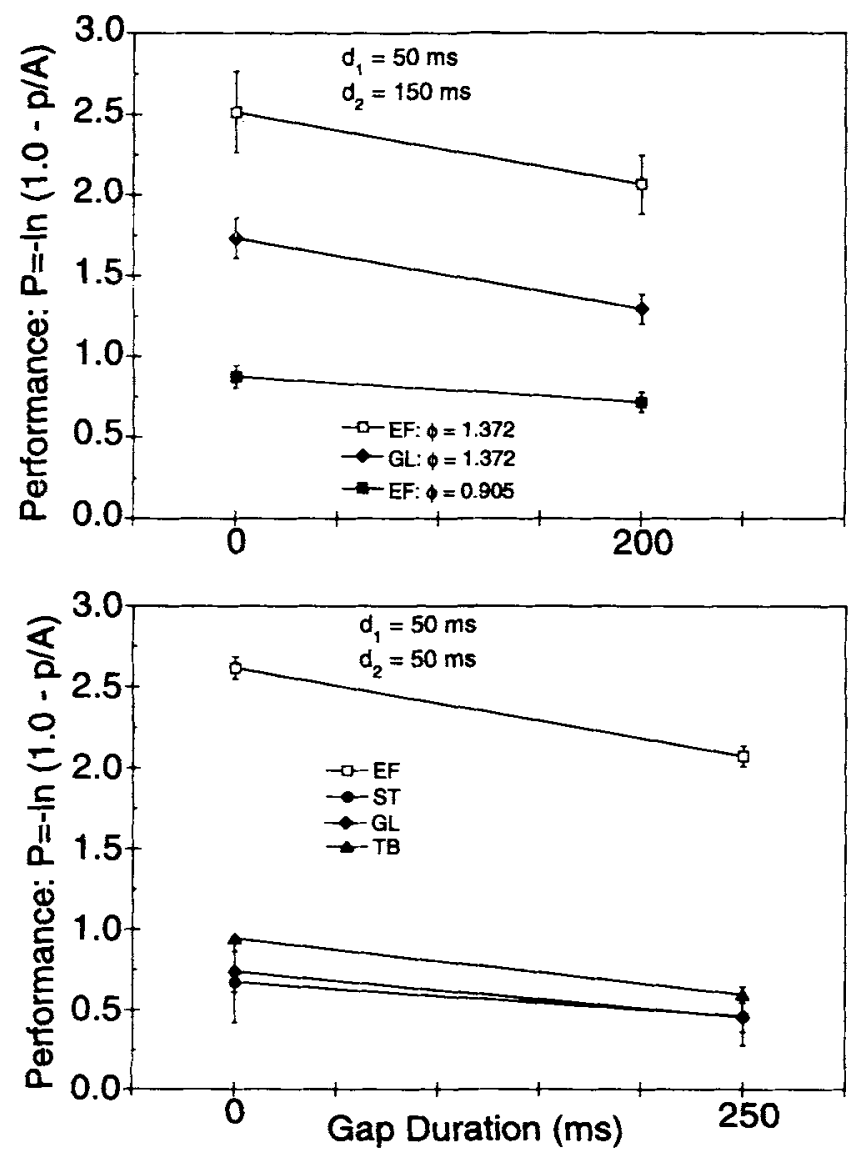

Figure 7. Experiment 2: Performance as a function gap size. The different panels represent two different first-half/gap/second-half configurations. Curves within each panel represent different observers or intensity levels. intensity and each of two observed durations-the duration $L$ required for above-chance performance and the additional duration required for performance to rise from chance to any criterion performance level--was approximately constant. In addition, the product of intensity and total time required to reach a criterion level was approximately constant. This regularity approximately confirms Bloch's Law as it is applied to memory performance for relatively complex visual stimuli. It also replicates and extends Turvey's (1973) finding that the product of intensity and duration determines the probability with which an alphanumeric stimulus will escape being masked.

The theory we used to account for these findings incorporated a front-end linear filter that operates on the stimulus intensity function to produce what we term a sensoryresponse function. The theory then assumes an informationacquisition process that, at any given time, is proportional to the product of (1) the magnitude by which the sensory response exceeds some threshold sensory response, and (2) the proportion of yet-to-be-acquired stimulus information. This five-parameter theory accounts quite well for the Experiment 1 data, predicting in the process the form of our durationintensity trade-offs that we found.

In Experiment 2, we tested and confirmed a particular prediction of this linear-response-with-threshold theory: that with brief, low-intensity displays, a stimulus presented once is recalled better than a stimulus presented for the same total time but broken into two temporally distinct parts.

\section{Bloch's Law, Perceptual Metamers, and "Memory Metamers"}

We now discuss the interrelations among our Experiment 1 results, Bloch's Law, and our theory. We begin by considering three stimulus pairs, each pair configured such that the duration $X$ intensity product is the same for each member of the pair. We refer to such stimuli as equal-product stimuli. Denoting a stimulus in terms of its intensity value $X$ duration value, the first pair is $2 \mathrm{~cd} / \mathrm{m}^{2} \times 10 \mathrm{~ms}$ and $1 \mathrm{~cd} / \mathrm{m}^{2} \times 20$ $\mathrm{ms}$; the second pair is $2 \mathrm{~cd} / \mathrm{m}^{2} \times 40 \mathrm{~ms}$ and $1 \mathrm{~cd} / \mathrm{m}^{2} \times 80$ $\mathrm{ms}$; and the third pair is $2 \mathrm{~cd} / \mathrm{m}^{2} \times 100 \mathrm{~ms}$ and $1 \mathrm{~cd} / \mathrm{m}^{2} \times$ $200 \mathrm{~ms}$. The $a(t)$ functions emerging from these six stimuli are shown in Figure 8. In each panel, the solid line corresponds to the shorter, more intense stimulus, and the dashed line corresponds to the longer, less intense stimulus.

When different stimuli lead to similar sensory-response functions. As noted by Watson (1986), the stimulus representations in Figure 8 suggest a parsimonious explanation for Bloch's Law: two equal-product stimuli will be detected with similar probability to the degree that they produce similar sensory-response functions. This explanation follows no matter what specific detection mechanism is hypothesized as long as detection occurs "downstream" from the sensoryresponse function (i.e., somewhere in the system where the only available information about the stimulus is based on the sensory-response function). This is because, by definition, any downstream part of the system cannot have more information about a stimulus than is contained in the stimulus's sensory-response function. Thus, in the extreme, if two 

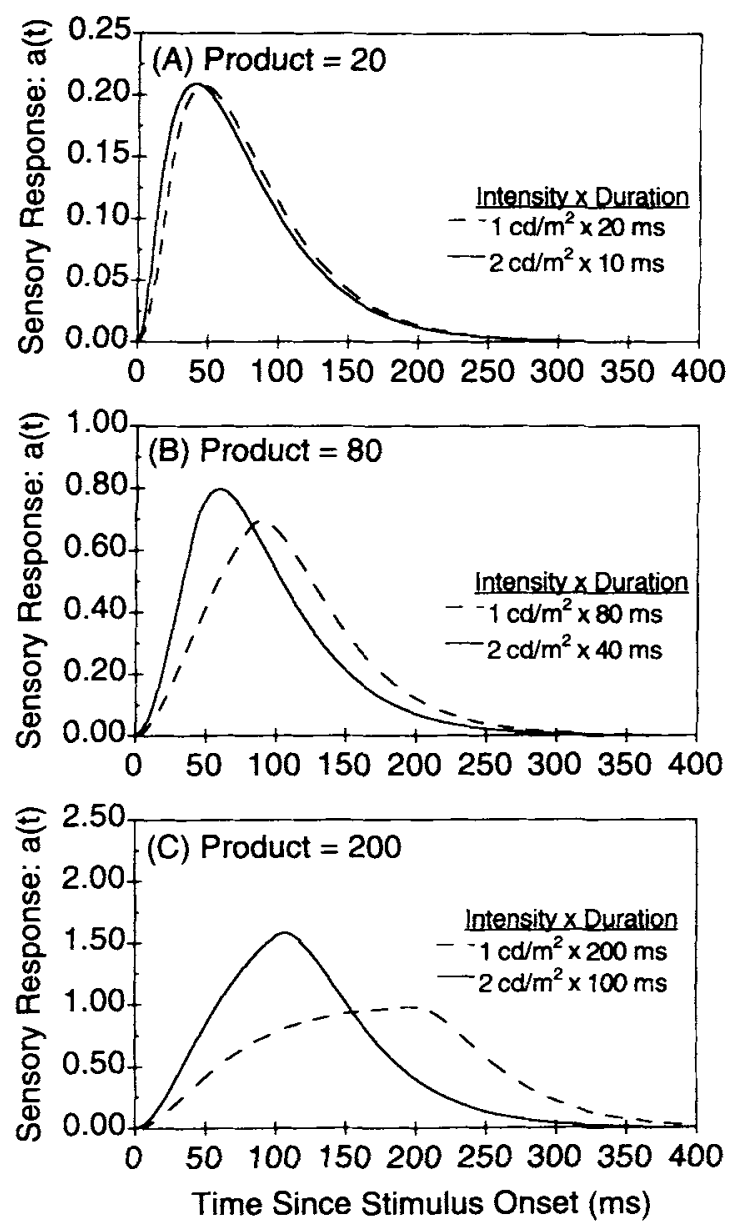

Figure 8. Three examples of sensory-response functions associated with equal-product stimulus pairs. In each panel, the solid line represents the shorter, more intense pair member, and the dashed line represents the longer, less intense pair member.

equal-product stimuli produced identical sensory-response functions (as is essentially the case with the Figure 8A stimuli), they would be indistinguishable by any possible test (including in particular any detection test) that is based on a downstream representation.

Metamers. In color vision, the term metameric is used to describe two stimuli composed of physically different wavelength mixtures that are perceived to be identical. In the present context, two stimuli producing the identical sensoryresponse function could similarly be termed metameric if, in general, metamers are defined to be physically different stimuli that produce identical responses at some peripheral stage of the perceptual-cognitive system.

So, for instance, the two Figure $8 \mathrm{~A}$ stimuli must lead to equal detection performance (or any other kind of performance) because they produce essentially identical responses at some presumably early stage. However, as equal-product stimuli get longer, the corresponding sensory-response functions become less similar: As indicated in Figures $8 \mathrm{~B}$ and $8 \mathrm{C}$, the longer member of the pair produces a longer, flatter function than does the shorter member. Thus, the longer a pair of equal-product stimuli, the more distinguishable become their sensory-response functions, and the easier it is for the system to devise a test that will distinguish them. In one class of detection models, for instance, detection occurs if the sensory-response function exceeds some threshold; thus, as illustrated in Figure 8, the longer the stimuli, the more probable it is that the shorter, brighter one will be detected in relation to the longer, dimmer one (and this is exactly what happens). This is a reasonable explanation of why Bloch's Law "breaks down" at long durations.

Memory metamers. Although it is possible for the perceptual-cognitive system to distinguish a shorter duration from a longer duration, equal-product stimulus, it does not follow that any representations generated by the system to such pairs can be distinguished. In particular, any stimulus representation that depends only on the area under the sensory-response function cannot serve as a basis for distinguishing any of the Figure 8 stimulus pairs, as the area under the two curves is identical in all three cases. The theory that we have presented supposes such representations; accordingly, the Bloch's Law-like effects observed in the data would, unlike real Bloch's Law effects, continue to hold with indefinitely long stimuli (see Kahneman \& Norman, 1964, and Wasserman \& Kong, 1979, for an additional discussion about why Bloch's Law applies in somewhat different ways to different tasks involving the same physical stimuli). Generalizing the notion of a metamer yet further, such stimulus pairs might be termed memory metamers: The two pair members would yield different perceptual experiences but identical memory representations. Perceptual metamers could, in contrast, be defined as stimuli, such as classical color metamers, that are indistinguishable by any stage of the perceptual-cognitive system.

\section{Information Extraction and Phenomenology}

These remarks bring us to our last topic: the link between the phenomenological appearance of some stimulus on the one hand, and the ultimate memory representation that issues from the stimulus on the other hand.

Essentially, we have argued that, while these two facets of perception and cognition are separable, and influenced by different variables, they are, within the context of our theory, determined by two facets of the same function: the sensoryresponse function. Roughly speaking, phenomenology is determined by the shape of the sensory-response function, while the memory representation is determined by the area under the sensory-response function.

This argument is aptly illustrated by results from another project in our laboratory (Loftus, Futhey, \& Russon, 1993) within which we carried out several modifications of the present Experiment 2. As in Experiment 2, we display stimuli of constant total physical duration in either a no-gap or a gap condition. However, instead of being simple digits tested by immediate recall, the stimuli were complex, naturalistic scenes, tested by delayed recognition. Of some importance 
is that these pictures were presented at much higher intensities than were the present digit stimuli. ${ }^{14}$ We reasoned that with high-intensity stimuli, the presumed sensory threshold would be low in relation to overall intensity and accordingly, any threshold-driven effect-such as the gap effect-would be substantially diminished. We discovered that there was no significant gap effect. Power analyses indicated that any actual gap effect could not have been greater than about $2 \%$ as gap size was increased from 0 to $250 \mathrm{~ms}$.

Thus, in accord with the predictions of the simplest (i.e., threshold-less) linear-filter model, the gap and no-gap conditions produced memory representations that we have just characterized as memory metamers. To determine whether the two conditions were perceptually distinguishable, we also asked observers to distinguish between gap and no-gap stimuli at the time of original viewing. Observers were able to make this distinction perfectly, which meant that the gap and no-gap conditions did not produce perceptual metamers. The ability to perceptually distinguish gap and no-gap stimuli destined to be indistinguishable in a later recognition test is quite understandable within the context of our theory. It is, in particular, a consequence of the gap and no-gap stimuli having two entirely differently shaped sensory-response functions (see Figure 6) that led to different (nonmetameric) sensory experiences, but with the same areas under the sensory-response functions, which led to identical (metameric) memory representations. In short, these two salient aspects of perception-phenomenological appearance and information acquisition-are united as two aspects of the same sensory-response function.

${ }^{14}$ Because the pictures involved different areas of different intensity and color, intensity could not be precisely measured. Roughly speaking, the contrast of a typical object in a typical picture, against a typical background, was an order of magnitude greater than the contrasts used in the present experiments.

\section{References}

Bundesen, C. (1990). A theory of visual attention. Psychological Review, 97, 523-547.

Busey, T. A., \& Loftus, G. R. (in press). Sensory and cognitive components of visual information acquisition. Psychological Review.

Clark, C. C., \& Hogben, J. H. (1991). Visible persistence following a brief increment in stimulus luminance. Perception and Psychophysics, 49, 212-226.

Di Lollo, V., \& Dixon, P. (1992). Is the icon's worth apples and oranges? Comments on Loftus, Duncan, and Gehrig (1992). Journal of Experimental Psychology: Human Perception and Performance, $18,550-555$.

Dixon, P., \& Di Lollo, V. (in press). Beyond visible persistence: An alternative account of temporal integration and segregation in visual processing. Cognitive Psychology.

Eriksen, C. W. (1980). The use of a visual mask may seriously confound your experiment. Perception and Psychophysics, 28, 89-92.

Groner, M. T., Bischof, W. F., \& Di Lollo, V. (1988). A model of visible persistence and temporal integration. Spatial Vision, 3, 293-304.
Kahneman, D. (1968). Method, findings, and theory in studies of visual masking. Psychological Bulletin, 70, 404-425.

Kahneman, D., \& Norman, D. (1964). The time-intensity relation in visual perception as a function of the observer's task. Journal of Experimental Psychology, 65, 215-220.

Kaswan, J., \& Young, S. (1963). Stimulus exposure time, brightness, and spatial factors as determinants of visual perception. Journal of Experimental Psychology, 65, 113-123.

Kowler, E., \& Sperling, G. (1980). Transient stimulation does not aid visual search: Implications for the role of saccades. Perception and Psychophysics, 27, 1-10.

Loftus, G. R. (1985). Picture perception: Effects of luminance level on available information and information-extraction rate. Journal of Experimental Psychology: General, 114, 342-356.

Loftus, G. R., \& Busey, T. A. (1992). Multidimensional models and iconic decay. Journal of Experimental Psychology: Human Perception and Performance, 18, 356-361.

Loftus, G. R., Busey, T. A., \& Senders, J. W. (1993). Providing a sensory basis for models of visual information acquisition. Manuscript submitted for publication.

Loftus, G. R., Duncan, J., \& Gehrig, P. (1992). On the time course of perceptual information that results from a brief visual presentation. Journal of Experimental Psychology: Human Perception and Performance, 18, 530-549.

Loftus, G. R., Futhey, E., \& Russon, R. (1993). Perceptual metamers and memory metamers. Unpublished manuscript, University of Washington, Psychology Department, Seattle.

Loftus, G. R., \& Hanna, A. M. (1989). The phenomenology of spatial integration: Data and models. Cognitive Psychology, 21, 363-397.

Loftus, G. R., Hanna, A., \& Lester, L. (1988). Conceptual masking: How one picture steals attention from another picture. Cognitive Psychology, 20, 237-282.

.Loftus, G. R., \& Hogden, J. (1988). Picture perception: Information extraction and phenomenological persistence. In G. H. Bower (Ed.), The psychology of learning and motivation (Vol. 22). San Diego, CA: Academic Press.

Loftus, G. R., \& Irwin, D. E. (1993). Visible and informational persistence: Different tasks measure different things. Manuscript submitted for publication.

Loftus, G. R., \& Kallman, H. J. (1979). Encoding and use of detail information in picture recognition. Joumal of Experimental Psychology: Human Learning and Memory, 5, 197-211.

Loftus, G. R., Kaufman, L., Nishimoto, T., \& Ruthruff, E. (1992). Why it's annoying to look at slides with the room lights still on: Effects of visual degradation on perceptual processing and longterm visual memory. In K. Rayner (Ed.), Eye movements and visual cognition: Scene perception and reading (pp. 203-226). New York: Springer-Verlag.

Massaro, D. W. (1970). Perceptual processes and forgetting in memory tasks. Psychological Review, 77, 557-567.

Nisly, S. J., \& Wasserman, G. S. (1989). Intensity dependence of perceived duration: Data, theories, and neural integration. Psychological Bulletin, 106, 483-496.

Potter, M. C., \& Levy, E. I. (1969). Recognition memory for a rapid sequence of pictures. Journal of Experimental Psychology, 81 10-15.

Raab, D., \& Fehrer, E. (1962). Supplementary Report: The effect of stimulus duration and luminance on visual reaction time. Journal of Experimental Psychology, 64, 326-327.

Rumelhart, D. E. (1969). A multicomponent theory of the perception of briefly exposed visual displays. Journal of Mathematical Psychology, 7, 191-218. 
Shaffer, W. O., \& Shiffrin, R. M. (1972). Rehearsal and storage of visual information. Journal of Experimental Psychology, 92, 292-295.

Shibuya, H., \& Bundesen, C. (1988). Visual selection from multielement displays: Measuring and modeling effects of exposure duration. Journal of Experimental Psychology: Human Perception and Performance, 14, 591-600.

Sperling, G. (1986). A signal-to-noise theory of the effects of luminance on picture memory: Commentary on Loftus. Journal of Experimental Psychology: General, 115, 189-192.

Stoddard, P. K., \& Loftus, G. R. (1988). An IBM XT-compatible, computer-based, slide-projector laboratory. Behavioral Research Methods, Instrumentation, and Computers, 20, 541-551.
Townsend, J. T. (1981). Some characteristics of visual whole report behavior. Acta Psychologica, 47, 149-173.

Turvey, M. T. (1973). On peripheral and central processes in vision: Inferences from an information-processing analysis of masking with patterned stimuli. Psychological Review, 80, 1-52.

Wasserman, G. S., \& Kong, K. L. (1979). Absolute timing of mental activities. The Behavioral and Brain Science, 2, 243-255.

Watson, A. B. (1986). Temporal sensitivity. In K. R. Boff, L. Kaufman, \& J. P. Thomas (Eds.), Handbook of perception and human performance (Vol. 1, pp. 6-1-6-43). New York: Wiley.

Zacks, J. L. (1970). Temporal summation phenomena at threshold: Their relation to visual mechanisms. Science, 170 (3954), 197199.

\section{Appendix A}

\section{Proof That Performance Is Monotonically Related to Area Under the $a(t)$ Function}

Information extraction rate $r(t)$ is the derivative of acquired information $I(t)$ with respect to time. Also, $r(t)$ is assumed to be the product of $a(t)$ and $h[I(t)$. Thus,

$$
r(t)=\frac{d[I(t)]}{d t}=a(t) h[I(t)]
$$

or

$$
\frac{d[I(t)]}{h[I(t)]}=a(t) d t .
$$

By integrating both sides of Equation $\mathrm{Al}$, we find that

$$
H[I(t)]=A(t)+k
$$

where $A(t)$ is the integral of $a(t), H[I(t)]$ is the integral of
$\{1 / h[I(t)]\}$, and $k$ is the constant of integration. When $t=0, A(t)$ $=0$, and $I(t)=0$; hence $k=H(0)$. Therefore,

$$
H[I(t)]=A(t)+H(0) .
$$

Because $H$ is an integral, it is monotonically increasing and has an inverse, $H^{-1}$, which is also monotonic. From Equation $\mathrm{A} 2$, we get

$$
I(t)=H^{-1}[A(t)+H(0)]
$$

or, when $t=\infty$,

$$
I(\infty)=H^{-1}[A(\infty)+H(0)]
$$

Therefore $I(\infty)$ is a monotonic function of $A(\infty)$. Because $p$ is assumed to be a monotonic function of $I(\infty)$, and $P$ is assumed to be a monotonic function of $p, P$ is a monotonic function of $A(\infty)$. This completes the proof.

\section{Appendix B}

\section{Linear Performance Curves Result From Assumptions That $h[I(t)]$ Is Linear and That $m$ Is the Identity Function}

Let $h[I(t)]=[1.0-I(t)] / c^{\prime}$, where $c^{\prime}$ is a constant. Substituting this into Equation A1 (Appendix A), we get

$$
\frac{d[I(t)]}{[1.0-I(t)]}=\frac{a(t) d t}{c^{\prime}}
$$

By integrating both sides of Equation Bl, we get

$$
-\ln [1-I(t)]=\frac{A(t)}{c}+k,
$$

where $k$ is the constant of integration. When $t=0, A(t)=0$, and $I(t)=0$; thus $k=0$, and

$$
-\ln [1-I(t)]=\frac{A(t)}{c^{\prime}}
$$

Because $p=I(\infty)$

$$
-\ln [1-p]=\frac{A(\infty)}{c^{\prime}}
$$

By substituting $P=-\ln (1-p)$ and $A(\infty)=F(\infty)=\phi d$, we get

$$
P=\frac{\phi d}{c^{\prime}}
$$

By letting $c$ be proportional to $1 / \phi$, or $c=(1 / \phi) c^{\prime}$, we get

$$
P=\frac{d}{c}
$$

This completes the proof. 


\title{
Appendix C
}

\author{
Proof That When $r(t)$ Is Proportional to the Magnitude by Which $a(t)$ Exceeds Threshold $a_{t}$, $P$ Is \\ Monotonically Related to Area Under $a(t)$ Above Threshold
}

We have already shown (Appendix A) than when $r(t)=$ $a(t) H[I(t)]$, performance $P$ is a monotonic function of $A(\infty)$. We define a new function,

$$
a_{t}(t)=a(t)-a_{t}
$$

and let $r(t)=a_{t}(t) H[I(t)]$, as assumed in the text. Then by the arguments in Appendix A,P must be a monotonic function of $A_{r}(\infty)$, the total area under $a_{t}(t)$. This is equal to the area under $a(t)$ that is above the threshold $a_{r}$.

Received August 31, 1992 Revision received February 23, 1993

Accepted March 1, 1993

\section{Instructions to Authors}

Authors should prepare manuscripts according to the Publication Manual of the American Psychological Association (3rd ed.). All manuscripts must include an abstract containing a maximum of 960 characters and spaces (which is approximately 120 words) typed on a separate sheet of paper. All copy must be double-spaced. Instructions for preparation of abstracts, tables, figures, references, and abbreviations of measurements appear in the Manual. All manuscripts are subject to editing for sexist language. For further information on content, authors may refer to an editorial by the previous editor, James Cutting, in the May 1988 issue of the Journal (Vol. 14, No. 2, p. 322). For information on the other three $J E P$ journals, authors should refer to editorials in those journals.

APA policy prohibits an author from submitting the same manuscript for concurrent consideration by two or more publications. In addition, it is a violation of APA Ethical Principles to publish "as original data, data that have been previously published" (Standard 6.24). As this journal is a primary journal that publishes original material only, APA policy prohibits as well publication of any manuscript that has already been published in whole or substantial part elsewhere. Authors have an obligation to consult journal editors concerning prior publication of any data upon which their article depends. In addition, APA Ethical Principles specify that "after research results are published, psychologists do not withhold the data on which their conclusions are based from other competent professionals who seek to verify the substantive claims through reanalysis and who intend to use such data only for that purpose, provided that the confidentiality of the participants can be protected and unless legal rights concerning proprietary data preclude their release" (Standard 6.25). APA expects authors submitting to this journal to adhere to these standards. Specifically, authors of manuscripts submitted to APA journals are expected to have available their data throughout the editorial review process and for at least 5 years after the date of publication.

Authors will be required to state in writing that they have complied with APA ethical standards in the treatment of their sample, human or animal, or to describe the details of treatment. A copy of the APA Ethical Principles may be obtained by writing the APA Ethics Office, 750 First Street, NE, Washington, DC 20002-4242.

Masked reviews are optional, and authors who wish masked reviews must specifically request them when submitting their manuscripts. Each copy of a manuscript to be mask reviewed should include a separate title page with authors' names and affiliations, and these should not appear anywhere else on the manuscript. Footnotes identifying the authors should be typed on a separate page. Authors should make every effort to ensure that the manuscript itself contains no clues to their identities.

Five copies of the manuscripts should be submitted. All copies should be clear, readable, and on paper of good quality. A dot matrix or unusual typeface is acceptable only if it is clear and legible. In addition to addresses and phone numbers, authors should supply electronic mail addresses and fax numbers, if available, for potential use by the editorial office and later by the production office. Authors should keep a copy of the manuscript to guard against loss. Mail manuscripts to the Editor, Thomas H. Carr, Department of Psychology, Michigan State University, East Lansing, Michigan 48824-1117.

For the other JEP journals, authors should submit manuscripts (in quadruplicate) to one of the editors at the following addresses: Journal of Experimental Psychology: General, Earl Hunt, Department of Psychology N1-25, University of Washington, Seattle, Washington 98195; Journal of Experimental Psychology: Learning, Memory, and Cognition, Keith Rayner, Department of Psychology, Tobin Hall, University of Massachusetts, Amherst, Massachusetts 01003; Journal of Experimental Psychology: Animal Behavior Processes, Stewart H. Hulse, Editor, Department of Psychology, Johns Hopkins University, Baltimore, Maryland 21218; and Journal of Experimental Psychology: Applied, Raymond S. Nickerson, Department of Psychology, Tufts University, Medford, Massachusetts 02155 . When one of the editors believes a manuscript is clearly more appropriate for an alternative journal of the American Psychological Association, the editor may redirect the manuscript with the approval of the author. 